\title{
Effective string theory description of the interface free energy
}

\author{
Marco Billó, Michele Caselle*, Livia Ferro \\ Dipartimento di Fisica Teorica, Università di Torino \\ and Istituto Nazionale di Fisica Nucleare - sezione di Torino, \\ Via P. Giuria 1, I-10125 Torino, Italy \\ E-mail: billo, caselle, ferro@to.infn.it
}

\section{Martin Hasenbusch}

Dipartimento di Fisica dell'Università di Pisa and I.N.F.N.,

Largo Bruno Pontecorvo 3, I-56127 Pisa, Italy

E-mail: Martin.Hasenbuschedf.unipi.it

\section{Marco Panero}

Institute for Theoretical Physics, University of Regensburg,

93040 - Regensburg, Germany

E-mail: marco.panero@physik.uni-regensburg

\begin{abstract}
We compare the predictions of the Nambu-Goto effective string model with a set of high precision Monte Carlo results for interfaces with periodic boundary conditions in the 3D Ising model. We compute the free energy in the covariant gauge exactly, up to the inclusion of the Liouville mode. The perturbative expansion of this result agrees both with the result evaluated several years ago by Dietz and Filk in the physical gauge and with a recent calculation with the Polchinski-Strominger action. We also derive the effective string spectrum which, because of the different boundary conditions, is very different from the well known one of Arvis. Taking into proper account the effective string corrections and exploiting some technical improvements in the simulations we obtain precise estimate of the amplitude ratios $\frac{T_{c}}{\sqrt{\sigma}}, \frac{m_{0++}}{\sqrt{\sigma}}$ and $\sigma \xi_{2 n d}^{2}$. We also discuss the behaviour of the effective string free energy in the dimensional reduction limit (i.e., near the deconfinement transition of the dual $3 \mathrm{~d}$ gauge Ising model) and its relationship with the $2 \mathrm{~d}$ Ising model interfaces
\end{abstract}

The XXV International Symposium on Lattice Field Theory

July 30 - August 42007

Regensburg, Germany

\footnotetext{
* Speaker.
} 


\section{Introduction}

The properties of interfaces in three-dimensional statistical systems have been a long-standing subject of research. In particular the interest of people working in the subject has been attracted by the so called "fluid" interfaces whose dynamics is dominated by massless excitations (for a review see for instance [1]). For this class of interfaces, thanks to the presence of long range massless modes, microscopic details such as the lattice structure of the spin model or the chemical composition of the components of the binary mixture become irrelevant and the physics can be rather accurately described by field theoretic methods.

A simple realization of these fluid interfaces is represented by $3 \mathrm{~d}$ spin models. These models in the low temperature phase in which their global symmetry is broken admit different vacua which, for a suitable choice of the boundary conditions, can occupy macroscopic regions and are separated by domain walls which behave as interfaces. For temperatures between the roughening and the critical one, interfaces are dominated by long wavelength fluctuations (i.e. they exactly behave as fluid interfaces).

An effective model widely used to describe a rough interface is the capillary wave model (CWM) [2,3]. Actually this model (which was proposed well before the Nambu-Goto papers [4, 5]) exactly coincides with the Nambu-Goto one, since it assumes an effective Hamiltonian proportional to the variation of the surface's area with respect to the classical solution.

Among the various realizations of fluid interfaces, a prominent role has been played in these last years by the $3 \mathrm{~d}$ Ising model, for several reasons. The universality class of the Ising model includes many physical systems, ranging from binary mixtures to amphiphilic membranes. It also shares the universality class with the $\phi^{4}$ theory; this allows a QFT approach to the description of the interface physics. Last but not least, the Ising model, due to its intrinsic simplicity, allows fast and high statistics Monte Carlo simulations, so that very precise comparisons can be made between theoretical predictions and numerical results.

Another important reason of interest of interfaces in the $3 \mathrm{~d}$ Ising model is that, thanks to the duality relation with the $3 \mathrm{~d}$ gauge Ising model, they are deeply related with Wilson loop observables. This is the ultimate reason for the validity of the Nambu-Goto effective string model for interfaces. Indeed this mapping allows to create a dictionary between Lattice Gauge Theory (LGT) observables and interface ones and makes the results obtained in the context of interface physics immediately relevant also for LGT studies. In this respect, it is important to stress that by working directly in the $3 \mathrm{~d}$ Ising model one has a larger freedom in the choice of the boundary conditions. In this work we shall exploit this freedom and address interfaces with periodic boundary conditions. This choice is particularly useful if one wants to address subtle finite size corrections (as one has to do in order to test the Nambu-Goto effective string picture) since the periodic b.c. allow to eliminate any source of boundary corrections in the results, thus leading to a very clean and artefact-free comparison between theory and simulations.

The aim of this contribution is to summarize a set of results recently obtained in this context by our group $[6,7,8,9]$. In particular we shall discuss the following items:

1] Explicit evaluation of the Nambu-Goto partition function (neglecting the anomaly) for interfaces [6]. 
2] A collection of numerical results from a set of high precision Montecarlo simulations $[7,8]$

3] The $2 \mathrm{~d}$ limit of $3 \mathrm{~d}$ interfaces [9].

\section{The partition function of interfaces from the Nambu-Goto effective string}

The Nambu-Goto bosonic string is defined by an action proportional, via the string tension $\sigma$, to the induced area of a surface embedded in a $d$-dimensional target space:

$$
S=\sigma \int d^{2} \xi \sqrt{\operatorname{det} g}, \quad g_{\alpha \beta}=\frac{\partial X^{i}}{\partial \xi^{\alpha}} \frac{\partial X^{j}}{\partial \xi^{\beta}} G_{i j}
$$

where the proper coordinates $\xi^{\alpha}$ parametrize the world sheet while $X^{i}(\xi)(M=0, \ldots, d)$ describes the target space position of a point specified by $\xi$. We shall assume in the following that the target space metric $G_{i j}$ is the flat one and neglect it.

The standard approach to evaluate the partition function is to fix the re-parametrizations of the action by fixing the so-called "physical" gauge in which the proper coordinates are identified with two of the target space coordinates, say $X^{0}$ and $X^{1}$. The quantum version of the NG theory can then be defined through the functional integration over the $d-2$ transverse d.o.f. $\vec{X}\left(X^{0}, X^{1}\right)$ of the gauge-fixed action. The partition function for a surface $\Sigma$ with prescribed boundary conditions is given by

$$
\begin{aligned}
Z_{\Sigma} & =\int_{(\partial \Sigma)} D X^{i} \exp \left\{-\sigma \int_{\Sigma} d X^{0} d X^{1}\left(1+\left(\partial_{0} \vec{X}\right)^{2}+\left(\partial_{1} \vec{X}\right)^{2}+\left(\partial_{0} \vec{X} \wedge \partial_{1} \vec{X}\right)^{2}\right)^{\frac{1}{2}}\right\} \\
& =\int_{(\partial \Sigma)} D X^{i} \exp \left\{-\sigma \int_{\Sigma} d X^{0} d X^{1}\left[1+\frac{1}{2}\left(\partial_{0} \vec{X}\right)^{2}+\frac{1}{2}\left(\partial_{1} \vec{X}\right)^{2}+\text { interactions }\right]\right\} .
\end{aligned}
$$

Expanding the square root as in the second line above, the classical area law $\exp (-\sigma \mathscr{A})$ (where $\mathscr{A}$ is the area of the minimal surface $\Sigma$ ) is singled out. It multiplies the quantum fluctuations of the fields $\vec{X}$, which have a series of higher order (derivative) interactions. The functional integration can be performed perturbatively (since we are studying an effective model we can neglect the fact that the theory is actually non-renormalizable), the loop expansion parameter being $1 /(\sigma \mathscr{A})$, and it depends on the boundary conditions imposed on the fields $\vec{X}$, i.e., on the topology of the boundary $\partial \Sigma$ and hence of $\Sigma$. The cases in which $\Sigma$ is a disk, a cylinder or a torus are the ones relevant for an effective string description of, respectively, Wilson loops, Polyakov loop correlators and interfaces in a compact target space. The computation was carried out up to two loops in [10].

The main problem of this approach is that the physical gauge is anomalous in $d \neq 26$ dimensions. However it can be shown [11] that the anomaly decreases as $R^{-3}$, where $R$ is the typical scale of the boundary of $\Sigma$ and thus it could be possible that it does not affect the first two terms in the loop expansion.

In [6] we proposed an alternative treatment of the NG model which takes advantage of the first order formulation, in which the action is simply

$$
S=\sigma \int d \xi^{0} \int_{0}^{2 \pi} d \xi^{1} h^{\alpha \beta} \partial_{\alpha} X^{i} \partial_{\beta} X^{i}
$$


where $h_{\alpha \beta}$ is an independent world-sheet metric, $\xi^{1} \in[0,2 \pi]$ parametrizes the spatial extension of the string and $\xi^{0}$ its proper time evolution. Integrating out $h$, we retrieve the NG action eq. (2.1). For each topology of the world-sheet, classified simply by its genus $g$, we can instead use re-parametrization and Weyl invariance to put the metric in a reference form $\mathrm{e}^{\phi} \hat{h}_{\alpha \beta}$ (conformal gauge fixing). For instance, on the sphere, i.e. at genus $g=0$, we can choose $\hat{h}_{\alpha \beta}=\eta_{\alpha \beta}$, while on the torus, at genus $g=1, \hat{h}_{\alpha \beta}$ is constant, but still depends on a single complex parameter $\tau$, the modulus of the torus. The scale factor $\mathrm{e}^{\phi}$ decouples at the classical level and the action takes then the form

$$
S=\sigma \int d \xi^{0} \int_{0}^{2 \pi} d \xi^{1} \hat{h}^{\alpha \beta} \partial_{\alpha} X^{i} \partial_{\beta} X^{i}+S_{\mathrm{gh} .},
$$

where $S_{\text {gh. }}$ in eq. (2.3) is the action for the ghost and anti-ghost fields (traditionally called $c$ and b) that arise from the Jacobian to fix the conformal gauge; we do not really need here its explicit expression, the only important thing to know is that they correspond to a CFT of central charge $c_{\text {gh. }}=-26$. The fields $X^{i}(\tau, \sigma)$, with $i=1, \ldots, d$, describe the embedding of the string world-sheet in the target space and form the simple, well-known two-dimensional CFT of $d$ free bosons. This allows to evaluate exactly the sum over all possible surfaces for any value of $d$. In the particular case of the toroidal geometry (interfaces) in which we are interested one finds (see [6] for the details):

$$
\mathscr{I}^{(d)}=2\left(\frac{\sigma}{2 \pi}\right)^{\frac{d-2}{2}} V_{T} \sqrt{\sigma \mathscr{A} u} \sum_{k, k^{\prime}=0}^{\infty} c_{k} c_{k^{\prime}}\left(\frac{E}{u}\right)^{\frac{d-1}{2}} K_{\frac{d-1}{2}}(\sigma \mathscr{A} E),
$$

where $\mathscr{A} \equiv L_{1} L_{2}$ denotes the minimal area of the interface, $u \equiv \frac{L_{2}}{L_{1}}$ is the ratio between the two lattice sizes of the plane in which the interface lies ( $L_{i}$ denotes the lattice sizes in the $i$ th direction), $V_{T}=\prod_{i=3}^{d} L_{i}$ is the transverse volume, $c_{k}$ is the $k$ th coefficient in the expansion of the Dedekind function:

$$
[\eta(q)]^{2-d}=\sum_{k=0}^{\infty} c_{k} q^{k-\frac{d-2}{24}}
$$

$K_{\frac{d-1}{2}}$ is the modified Bessel function of order $\frac{d-1}{2}$ and

$$
E=\sqrt{1+\frac{4 \pi u}{\sigma \mathscr{A}}\left(k+k^{\prime}-\frac{d-2}{12}\right)+\frac{4 \pi^{2} u^{2} n_{1}^{2}}{(\sigma \mathscr{A})^{2}}} .
$$

denotes the spectrum of the NG string for this particular type of geometry.

It is important to stress that also in this case for $d \neq 26$ we must expect deviations from this result. In fact the decoupling of the scale factor $\mathrm{e}^{\phi}$ persists at the quantum level only if the anomaly parametrized by the total central charge $c=d-26$ vanishes; Weyl invariance is otherwise broken and the mode $\phi$ has to be thought of as a field with a Liouville-type action.

Notwithstanding this there are a few non-trivial results of this analysis which are not affected by the anomaly and deserve further attention:

- Eq.(2.5) gives an explicit prediction for the functional dependence on $\sigma, \mathscr{A}, u$ and $V_{T}$ of the prefactor in the interface free energy which (as we shall see in the next section) nicely agrees with the results of the MC simulations. 

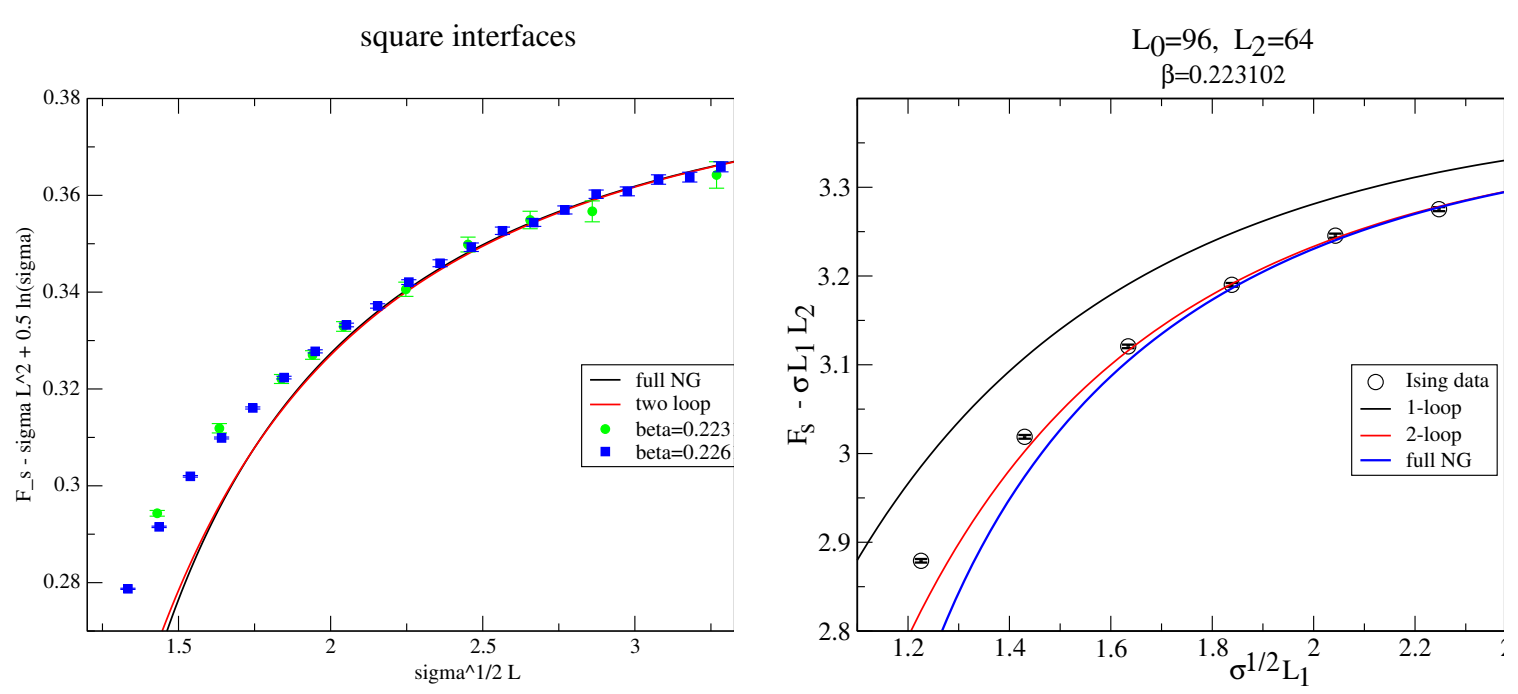

Figure 1: Fig.1a: Comparison of the 2-loop prediction, the full Nambu-Goto result with the data for square interfaces at $\beta=0.223102$ and $\beta=0.226102$. Fig.1b: $F_{\mathrm{s}}^{(2)}-\sigma L_{1} L_{2}$ as a function of $\sqrt{\sigma} L_{1}$ for $\beta=$ $0.223102, L_{3}=96$ and $L_{2}=64$. Note that in the case of the Monte Carlo results the statistical error is smaller than the symbol (circle). The 1-loop, 2-loop and full NG predictions are given as solid black, red and blue lines, respectively.

- The spectrum reported in eq.(2.7) is different from the one obtained by Arvis in [12] for the Wilson loop geometry and agrees with an independent calculation mentioned in [13].

- Eq.(2.5) in the $d=3$ case allows a smooth dimensional reduction limit and suggest an expression for the $2 \mathrm{~d}$ interface which agrees with an exact $2 \mathrm{~d}$ calculation.

- Expanding eq.(2.5) in powers of $1 /(\sigma \mathscr{A})$ we reobtain at the first two orders the same contributions obtained in [10] with the physical gauge. This is probably the most interesting result since it supports the idea that not only the first term in the expansion (the so called "Lüscher term" [14]) but also the next to leading order could be anomaly free and universal. This conjecture is further supported by the recent observation $[15,16]$ that the same term also appears in the expansion of the Polchinski-Strominger action [17] which is essentially a non-local, but anomaly free, version of the N-G action.

\section{Monte Carlo simulations of interfaces in the three-dimensional Ising model}

In order to test the above results it is mandatory to have very precise estimates of the interface 
free energy for different values of $L_{1}$ and $L_{2}$. In [7] and [8] we obtained these estimates using two different approaches. In [7] we used the "boundary flip" algorithm [18] which is particularly suited for interfaces of small or intermediate size while in [8] we obtained the interface free energy by a direct integration of the differences $E_{a}-E_{p}$ over $\beta$ (where $E_{a}$ denotes the internal energy for antiperiodic boundary conditions, and hence in presence of an odd number of interfaces, while $E_{p}$ denotes the internal energy with periodic b.c.). This second method is much more cumbersome from a computational point of view, but it is mandatory if one is interested in large values of the interface free energy. We refer to the original papers for all the details on the algorithms and on the simulation settings and only list here the main results.

- As it was already observed in the case of Wilson loop [19] and of Polyakov loop correlators [20], we found a very good agreement for large values of $R$ and a sizeable disagreement as $R$ decreases (see fig.1a). This deviation (due to the peculiar geometry of the interface observable) cannot be due to boundary corrections and is thus an intrinsic feature of the effective string description. In the case of square interfaces (i.e. $L_{1}=L_{2} \equiv R$ ) the NG effective string picture breaks down for values of $R$ smaller than $R \sim 2.5 / \sqrt{\sigma}$ (see fig.1a). It is important to notice that for square interfaces we see essentially no difference between the prediction obtained using eq.(2.5) (black line in fig.1a) and the two loop approximation (red line in fig.1a).

- In order to disentangle between the two predictions one must study asymmetric interfaces because higher orders in eq.(2.5) become more and more relevant as $u=L_{2} / L_{1}$ increases. This is evident in fig.1b where the two curves are well separated. Looking at fig.1b we see that the MC data show a much better agreement with the two loop approximation than with eq.(2.5). This deviation from the behaviour of eq.(2.5 may simply denote a breaking of the effective string picture in this asymmetric limit. However it is also possible (and the persistent agreement with the two loop approximation could support this interpretation) that the effective string picture still holds but the corrections due to the anomaly (or equivalently, in the covariant gauge framework, to the Liouville field) which affect the calculation of eq.(2.5) cannot be neglected.

- Last, but not least, thanks to the improvement in the estimate of the interface string tension $\sigma$ we could obtain new predictions for a few amplitude ratios which are more precise than any other existing estimate in the literature:

$$
\begin{gathered}
\frac{T_{c}}{\sqrt{\sigma}}=1.235(2) \\
\frac{m_{0++}}{\sqrt{\sigma}}=3.037(16) \\
R_{-} \equiv \sigma \xi_{2 n d}^{2}=0.1024(5)
\end{gathered}
$$




\section{Universal behaviour of interfaces in $2 \mathrm{~d}$ and dimensional reduction of Nambu-Goto string}

It is interesting to study the $u \rightarrow \infty$ (i.e. $L_{1}<<L_{2}$ ) limit of the eq.(2.5) for $d=3$. This corresponds to perform a dimensional reduction from $d=3$ to $d=2$ and allows us to obtain an effective description for bidimensional interfaces. The result is [9]:

$$
Z=\left(\frac{2}{\pi}\right)^{\frac{1}{2}} L_{3} m_{\mathrm{eff}} K_{1}\left(m_{\mathrm{eff}} L_{2}\right), \quad m_{\mathrm{eff}}=\sigma L_{1} \sqrt{1-\frac{\pi}{3 \sigma L_{1}^{2}}}
$$

Eq.(4.1) is most probably an exact result for any $2 \mathrm{~d}$ spin model and not just an effective approximation. Indeed it agrees with an exact calculation in the $2 \mathrm{~d}$ Ising model. Moreover it arises from a simple universal model for $2 \mathrm{~d}$ interfaces, based on the first order treatment of an action given by the length of the interface.

Acknowledgements: M.P. acknowledges support from the Alexander von Humboldt Foundation.

\section{References}

[1] V. Privman, Int. J. Mod. Phys. C 3 (1992) 857 [arXiv:cond-mat/9207003].

[2] F. P. Buff, R. A. Lovett and F. H. Stillinger Jr., Phys. Rev. Lett. 15 (1965) 621.

[3] J. Rowlinson and S. Widom, Molecular theory of capillarity, Clarendon Press, 1982.

[4] T. Goto, Prog. Theor. Phys. 46, 1560 (1971).

[5] Y. Nambu, Phys. Rev. D 10, 4262 (1974).

[6] M. Billó, M. Caselle and L. Ferro, JHEP 0602 (2006) 070 [arXiv:hep-th/0601191].

[7] M. Caselle, M. Hasenbusch and M. Panero, JHEP 0603 (2006) 084 [arXiv:hep-lat/0601023].

[8] M. Caselle, M. Hasenbusch and M. Panero, arXiv:0707.0055 [hep-lat].

[9] M. Billó, M. Caselle and L. Ferro, arXiv:0708.3302 [hep-th].

[10] K. Dietz and T. Filk, Phys. Rev. D 27 (1983) 2944.

[11] P. Olesen, Phys. Lett. B 160 (1985) 144.

[12] J. F. Arvis, Phys. Lett. B 127 (1983) 106.

[13] J. Kuti, PoS LAT2005 (2005) 001 [arXiv:hep-lat/0511023].

[14] M. Lüscher, K. Symanzik and P. Weisz, Nucl. Phys. B173 (1980) 365.

[15] J. M. Drummond, arXiv:hep-th/0411017.

[16] N. D. Hari Dass and P. Matlock, arXiv:hep-th/0612291.

[17] J. Polchinski and A. Strominger, Phys. Rev. Lett. 67 (1991) 1681.

[18] M. Hasenbusch, J.Phys. I (France) 3 (1993) 753, [arXiv:hep-lat/9209016].

[19] M. Caselle, R. Fiore, F. Gliozzi, M. Hasenbusch and P. Provero, Nucl. Phys. B 486 (1997) 245 [arXiv:hep-lat/9609041].

[20] M. Caselle, M. Hasenbusch and M. Panero, JHEP 0405 (2004) 032 [arXiv:hep-lat/0403004]. 